\title{
Quantitative Evaluation of Spinodal Decomposition in Fe-Cr by Atom Probe Tomography and Radial Distribution Function Analysis
}

\author{
Jing Zhou, ${ }^{1, *}$ Joakim Odqvist, $^{1,2}$ Mattias Thuvander, ${ }^{3}$ and Peter Hedström ${ }^{1}$ \\ ${ }^{1}$ Department of Material Science and Engineering, KTH Royal Institute of Technology, SE-10044 Stockholm, Sweden \\ ${ }^{2}$ AB Sandvik Materials Technology, R\&D Centre, SE-811 81 Sandviken, Sweden \\ ${ }^{3}$ Department of Applied Physics, Chalmers University of Technology, SE-412 96 Göteborg, Sweden
}

\begin{abstract}
Nanostructure evolution during low temperature aging of three binary Fe-Cr alloys has been investigated by atom probe tomography. A new method based on radial distribution function (RDF) analysis to quantify the composition wavelength and amplitude of spinodal decomposition is proposed. Wavelengths estimated from RDF have a power-law type evolution and are in reasonable agreement with wavelengths estimated using other more conventional methods. The main advantages of the proposed method are the following: (1) Selecting a box size to generate the frequency diagram, which is known to generate bias in the evaluation of amplitude, is avoided. (2) The determination of amplitude is systematic and utilizes the wavelength evaluated first to subsequently evaluate the amplitude. (3) The RDF is capable of representing very subtle decomposition, which is not possible using frequency diagrams, and thus a proposed theoretical treatment of the experimental RDF creates the possibility to determine amplitude at very early stages of spinodal decomposition.
\end{abstract}

Key words: atom probe tomography (APT), spinodal decomposition, radial distribution function (RDF), phase separation, stainless steels

\section{INTRODUCTION}

Spinodal decomposition is an important phase transformation in many technically relevant material systems. For instance, spinodal decomposition of TiAlN coatings may improve the performance of cutting tools due to enhanced hardness caused by the decomposition (Hörling et al., 2005). On the other hand, spinodal decomposition in ferritecontaining stainless steels, e.g., duplex steels, is believed to cause embrittlement (Chung \& Chopra, 1988; Chung \& Leax, 1990; Brown \& Smith, 1991; Zhou et al., 2012). The theory of spinodal decomposition originates from the research of Hillert (1956, 1961), Cahn and Hilliard (1958), and Cahn (1961). To verify the theory through experiments, Mössbauer spectroscopy (Chandra \& Schwartz, 1971), transmission electron microscopy (TEM) (Okada et al., 1978), atom probe field ion microscopy (APFIM) (Miller et al., 1982), small-angle neutron scattering (SANS) (Bley, 1992), and atom probe tomography (APT) (Miller et al., 1995) were gradually introduced to improve the understanding of spinodal decomposition in the Fe-Cr system. By the help of Mössbauer spectroscopy and TEM, spinodal decomposition can be detected, but it is difficult to determine the wavelength and amplitude, which are considered to be the two most important parameters in the assumed sinusoidal concentration variation in the theory of spinodal decomposition (Cahn, 1968). With the development of APT, quantitative measurements of spinodal decomposition were realized,

Received September 24, 2012; accepted February 13, 2013

*Corresponding author. E-mail: jingzhou@kth.se and the $\mathrm{Fe}-\mathrm{Cr}$ system was frequently analyzed because it provides a good model and has high technical relevance. Several different methods have been developed to measure the wavelength and amplitude of spinodal decomposition separately. There are, however, significant challenges associated with this quantitative analysis. For example, there are significant differences in amplitudes estimated using different methods - the selection of box size to evaluate local concentrations is critical, and the early stages of decomposition are very difficult to address because it is even difficult to observe a difference in, for example, the frequency diagram representation. One method that has been demonstrated particularly useful for representing the early stages of decomposition is the radial distribution function (RDF) (Thuvander et al., 2012; Zhou et al., 2012). However, so far no attempts to use the RDF to quantify spinodal decomposition have been reported. Thus, in the present work we make such an effort to evaluate both wavelength and amplitude during spinodal decomposition from RDF analysis. The RDF method is demonstrated for APT measurements on three binary Fe-Cr alloys after aging treatments, and the RDF method is evaluated versus other methods to quantify spinodal decomposition.

\section{Methods for Quantifying Spinodal DECOMPOSITION}

As mentioned, several methods for evaluating wavelength and amplitude of spinodal decomposition separately are available. This section is intended to provide a brief over- 
Table 1. Chemical Composition of the Three Investigated Binary Fe-Cr Alloys (wt\%).

\begin{tabular}{|c|c|c|c|c|c|c|c|c|c|}
\hline & $\mathrm{Cr}$ & $\mathrm{C}$ & $\mathrm{Si}$ & $\mathrm{Mn}$ & $S$ & $\mathrm{P}$ & $\mathrm{N}$ & $\mathrm{Ni}$ & $\mathrm{Fe}$ \\
\hline A & 25.28 & 0.002 & 0.09 & 0.09 & 0.006 & 0.004 & 0.009 & 0.03 & Bal. \\
\hline B & 30.42 & 0.004 & 0.06 & 0.11 & 0.008 & 0.006 & 0.006 & 0.02 & Bal. \\
\hline $\mathrm{C}$ & 36.10 & 0.005 & 0.27 & 0.09 & 0.005 & 0.005 & 0.008 & 0.02 & Bal. \\
\hline
\end{tabular}

view of some of these methods. For a more comprehensive view, the reader is referred to Miller (2000).

\section{Wavelength}

The auto-correlation function (ACF), equation (1), is an effective method to evaluate the wavelength from onedimensional (1D) concentration fields obtained using APFIM (Brenner et al., 1984; Oehring \& Alvensleben, 1988).

$$
R_{k}=\frac{1}{\sigma^{2}} * \sum_{i=0}^{N-k}\left(C_{i}-C_{0}\right)\left(C_{i+k}-C_{0}\right)
$$

where $C_{i}$ and $C_{i+k}$ are the concentration of the $i^{\prime}$ th and $i+k^{\prime}$ th sample blocks, $C_{0}$ is the average composition; $k$ is the lag, $N$ is the total number of sample blocks, and $\sigma^{2}$ is the variance of the composition of $C_{i}$, given by $\sigma^{2}=$ $\sum_{i=1}^{N}\left(C_{i}-C_{0}\right)^{2}$. Hyde et al. (1994) further extended the ACF method to also consider three-dimensional (3D) data. Recently, the RDF and fast Fourier transformation (FFT) analyses to evaluate wavelength were proposed in Odqvist et al. (2012), and the methods were evaluated versus ACF analysis. They found that the RDF method gave wavelengths in reasonable agreement with both methods, but the wavelength from RDF was found to be slightly larger than for the 1D methods in all cases.

\section{Amplitude}

To evaluate the amplitude, the definition of amplitude must first be considered. The two standard definitions are peakto-average composition for symmetrical distributions, i.e., sinusoidal concentration variations, and peak to trough. Here, peak to trough is chosen as the definition of amplitude. The Langer-Bar-on-Miller (LBM) method (Langer et al., 1975; Miller, 2000) is the most commonly used method to evaluate the amplitude. LBM is based on the frequency diagrams of $\mathrm{Cr}$ that can be described by two Gaussian curves, and that the two Gaussian peaks indicate the peak and trough composition, respectively. The LBM method is expressed by equation (2):

$$
\begin{aligned}
\rho(x)= & \frac{1}{\left(2 \pi * \sigma^{2}\right)^{1 / 2}} \\
& *\left\{\frac{\mu_{2}}{\mu_{1}+\mu_{2}} * \exp \left(-\left(x-\mu_{1}\right)^{2} / 2 \sigma^{2}\right)\right. \\
& \left.\quad+\frac{\mu_{1}}{\mu_{1}+\mu_{2}} * \exp \left(-\left(x-\mu_{2}\right)^{2} / 2 \sigma^{2}\right)\right\},
\end{aligned}
$$

where $\mu_{1}$ and $\mu_{2}$ are the peak and trough compositions, respectively, and $\sigma^{2}$ is the variance of the Gaussian distributions. The amplitude is $\mu_{2}-\mu_{1}$.
Another common method for evaluating the amplitude is the $P_{a}$ method (Sassen et al., 1987; Godfrey et al., 1988; Brown et al., 1990), which assumes that the concentration field has a sinusoidal distribution:

$$
x_{j}=C_{0}-P_{a} * \sin \frac{2 \pi j}{m},
$$

where $C_{0}$ is the average composition, $2 P_{\alpha}$ is the spinodal amplitude, $m$ is the discretization number, and $j$ is an integer from 1 to $m$.

Another example of a method to evaluate amplitude is the Variation method (Blavette et al., 1988) where the principle is to evaluate the difference between the experimental frequency distribution and the binomial distribution. This may be a good way to evaluate trends of decomposition but gives no quantitative measure of amplitude (Miller et al., 1992). It should be noted that all three methods explained in this section depend on the chosen block size to determine the concentration.

\section{Methodology}

\section{Materials and Heat Treatment}

Three binary Fe-Cr alloys were produced by vacuum arc melting, followed by homogenization at $1100^{\circ} \mathrm{C}$ for $2 \mathrm{~h}$ under pure argon, and a final quench in brine. The alloy designations and compositions are listed in Table 1. Thereafter, the alloys were aged at $500^{\circ} \mathrm{C}$ for $100 \mathrm{~h}$ and $1000 \mathrm{~h}(\mathrm{~A})$, $20 \mathrm{~h}$ and $200 \mathrm{~h} \mathrm{(B)}$, and $10 \mathrm{~h}$ and $100 \mathrm{~h}(\mathrm{C})$. One specimen per material condition was analyzed by APT. For more experimental details, see Xiong et al. (2011).

\section{Atom Probe Tomography}

The analyses were performed using a local electrode atom probe (LEAP 3000X HR ${ }^{\mathrm{TM}}$, Cameca, Madison, WI, USA), equipped with a reflectron for improved mass resolution. The ion detection efficiency is about $37 \%$, and the experiments were performed in voltage pulse mode $(20 \%$ pulse fraction, $200 \mathrm{kHz}$, evaporation rate $1.5 \%$ ) at $55 \mathrm{~K}$.

From the experimental raw data, 3D reconstructions were generated using the IVAS 3.4.3 software (Cameca). Hereby the following parameters were used: evaporation field $33 \mathrm{~V} / \mathrm{nm}$, field factor $\left(k_{f}\right) 3.8$, and image compression factor 1.8. It should be noted that $k_{f}$ is not known exactly, but it can be determined in case plane distances at identified crystallographic poles (Gault et al., 2008) can be measured, but that was not possible here. Therefore, the shape of the analyzed volume is somewhat uncertain. Brenner et al. (1982) reported that the spinodal decomposition for 
Fe-32 at.\% Cr appeared isotropic, and in the present work it was decided to use an isotropicity criterion to determine $k_{f}$. The $k_{f}$ was varied to give a structure that did not appear compressed or stretched in the $z$-direction. Furthermore, the voxel size used as the sampling size to generate the iso-concentration surfaces was $1 \mathrm{~nm} \times 1 \mathrm{~nm} \times 1 \mathrm{~nm}$.

\section{Radial Distribution Function Analysis}

RDF analysis was employed to represent the radial concentration profile starting from each and every detected atom of the chosen element. Thus it indicates the probability density of finding an atom $j$ at $r$ when an atom $i$ is the origin (Miller \& Kenik, 2004; De Geuser et al., 2006). In the RDFs presented here, $0.2 \mathrm{~nm}$ was taken as the step size and the shell thickness. The measured concentration at each position was normalized with respect to the average bulk concentration. The RDF can be expressed as

$$
\operatorname{RDF}(r)=\frac{C_{E}(r)}{C_{0}}=\frac{N_{E}(r) / N(r)}{C_{0}},
$$

where $C_{E}(r)$ is the atomic composition of element $\mathrm{E}$ at the distance of $r, C_{0}$ is the average composition of element $\mathrm{E}$ in the analyzed volume, $N_{E}(r)$ is the total number of atoms of element $\mathrm{E}$ at the distance of $r$, and $N(r)$ is the total number of atoms of all elements at the distance of $r$.

In RDF analysis of spinodal decomposition, the first maximum indicates the statistically closest distance between two Cr-rich regions and thus represents the wavelength. When no maximum can be found on the RDF curve due to small decomposition and statistical smoothing, it may still be possible to determine the wavelength by only generating the RDFs from a limited number of $\mathrm{Cr}$ atoms located in Cr-rich areas. This will be exemplified later in the Wavelength section.

To evaluate amplitude as well from RDF, one could think that if only the Cr-rich domains are chosen as the center to generate the $\mathrm{Cr}-\mathrm{Cr} \mathrm{RDF}$ curve, the first point could possibly be considered as the peak of the Cr composition profile. On the other hand, when all the Fe-rich regions are chosen as the center to generate the RDF curve of $\mathrm{Fe}-\mathrm{Cr}$, the first point could possibly be considered as the trough of the $\mathrm{Cr}$ composition profile. The crucial factor in such analysis of the amplitude is the selection of Cr-rich and Fe-rich domains, which can represent the peak and trough compositions. If too many $\mathrm{Cr}$-rich and Fe-rich regions are chosen, the amplitude will be underestimated; on the contrary, if too few Cr-rich and Fe-rich regions are chosen, the amplitude will be overestimated. This may seem trivial, but the key is to define the composition threshold in a systematic way to obtain a predefined number of domains. The wavelength obtained earlier can be used for this purpose. Assuming that each Cr-rich region is located in the center of a cube with the side length corresponding to the respective wavelength of spinodal decomposition and that all of the cubes are positioned side by side, the number of Cr-rich domains can be approximated by the following equation:

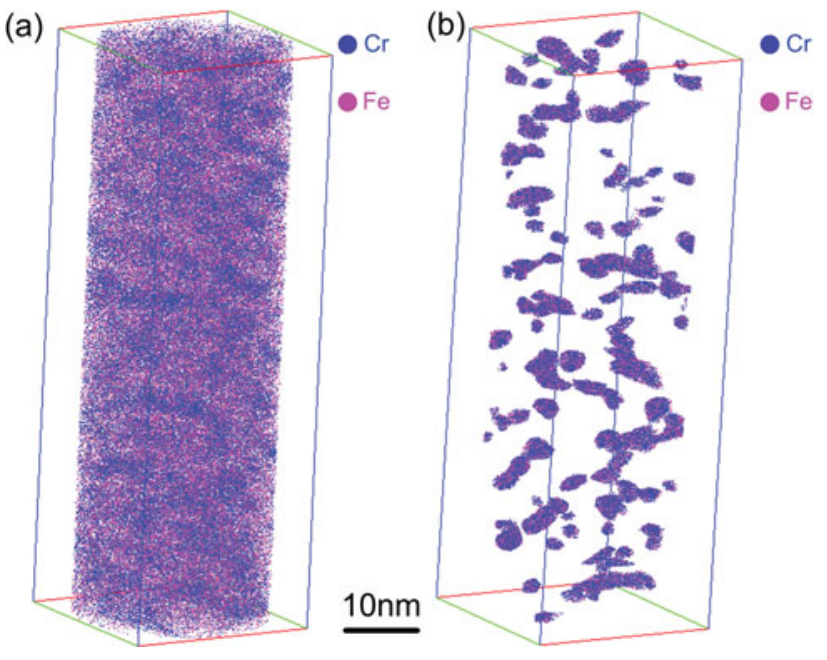

Figure 1. Atom maps for material A, aged for $1000 \mathrm{~h}$. a: The selected cylinder of $80 \mathrm{~nm} \times 27 \mathrm{~nm}$ used for general RDF analysis for all material conditions. b: Selected Cr-rich domains used to generate RDFs for subsequent determination of the peak composition.

$$
N=\frac{V}{\lambda^{3}}
$$

where $N$ is the total number of $\mathrm{Cr}$-rich or Fe-rich regions, $V$ is the total APT probed volume, and $\lambda$ is the wavelength of spinodal decomposition obtained by RDF analysis.

To calculate the number of $\mathrm{Cr}$-rich or Fe-rich regions easily, a fixed cylinder with the height of $80 \mathrm{~nm}$ and diameter of $27 \mathrm{~nm}$ was selected from each probed volume. In the IVAS software, iso-concentration surfaces were used to select the Cr-rich or Fe-rich regions. One example of such a selection of Cr-rich domains is presented in Figure 1. The atom map of $\mathrm{Cr}$ for one selected cylinder is shown in Figure 1a, and the selected Cr-rich domains are shown in Figure $1 \mathrm{~b}$. For this sample the predefined number of Crrich domains given by equation (5) was 96 , and by selecting an iso-concentration surface threshold of 46.2 at.\% Cr, a similar number of Cr-rich domains was obtained (Fig. 1b). From the selected Cr-rich domains, an RDF curve was generated to evaluate the peak composition, and similarly the Fe-rich domains were selected and the RDF of Fe-Cr was used to evaluate the trough.

Moreover, to try and rationalize the determination of amplitude from a theoretical perspective, it seemed important to derive an expression for the RDF curves in the present work. According to Cahn (1961), the interconnected spinodal structure is assumed to have a sinusoidal concentration variation for early stages of spinodal decomposition. The RDF value at distances approaching zero is related to the amplitude of this periodic concentration variation. The concentration is described by a periodic function $f(x, y, z)$ with wavelength $\lambda$. The probability of finding a $\mathrm{Cr}$ atom at the position of $\vec{r}(x, y, z)$ in space is expressed as $f(\vec{r})$. To generate the $\mathrm{RDF}$ curve, the concentration of $\mathrm{Cr}$ at the distance $r_{0}$ from the Cr at $\vec{r}(x, y, z)$ should be $\int_{\left|\vec{r}^{\prime}\right|=r_{0}} f(\vec{r}) *$ $f\left(\vec{r}+\vec{r}^{\prime}\right) d \vec{r}^{\prime}$. However, $f(\vec{r})$ should first be normalized [as 
in equation (4)] by dividing with the average composition $\left(C_{0}\right)$. Considering the zero distance for the RDF curve, $r_{0}$ should be $\boldsymbol{0}$. Then it should be integrated in one period of the sinusoidal distribution and normalized by the wavelength cube, i.e., one sinusoidal period in 3D. Using Cartesian coordinates $(x, y, z)$ the $\mathrm{RDF}(0)$ is given by

$$
\operatorname{RDF}(0)=\frac{1}{\lambda^{3}} \iiint_{x, y, z=-\lambda / 2}^{x, y, z=\lambda / 2}\left(\frac{f(x, y, z)}{C_{0}}\right)^{2} d x d y d z,
$$

where $f(x, y, z)=C_{0}+A \sin ((2 \pi / \lambda)(x+y+z))$ according to Cahn (1961).

It is then found that $\operatorname{RDF}(0)=1+A^{2} 2 C_{0}^{2}$. Furthermore, the amplitude of spinodal decomposition is obtained by

$$
2 A=2 C_{0} \sqrt{2(\operatorname{RDF}(0)-1)},
$$

where the amplitude is defined as two times " $\mathrm{A}$ " from peak to trough. Since the first point of the RDF, i.e., $\operatorname{RDF}(0)$, is uncertain according to Zhou et al. (2012), $\mathrm{RDF}(0)$ is determined by extrapolating the RDF curve to zero distance.

\section{Methods Used for Comparison with RDF}

In the present work, ACF analysis was used as comparison with the wavelength determinations from RDF. A cube with a size of $20 \mathrm{~nm} \times 20 \mathrm{~nm} \times 20 \mathrm{~nm}$ was extracted from the whole volume, and a $1 \mathrm{D}$ concentration profile was calculated by moving a $1 \mathrm{~nm} \times 1 \mathrm{~nm} \times 1 \mathrm{~nm}$ cube in the $x$-direction and stepping through the whole volume in the $y$ - and $z$-directions. A step size of $0.25 \mathrm{~nm}$ in the $x$-direction was selected to reconstruct the $1 \mathrm{D}$ concentration profiles. It should be pointed out that the step size can affect the wavelength obtained from ACF analysis, and in case a step size of $1 \mathrm{~nm}$ (same as the cube size) is used, the wavelength is seriously overestimated to about $15 \mathrm{~nm}$ for material C aged for $100 \mathrm{~h}$. This is obviously incorrect as compared to, for example, Brenner et al. (1984).

The LBM method was employed to compare with the amplitudes evaluated by RDF. According to Hetherington and Miller (1989), the results from LBM highly depend on the chosen block size, and this was evaluated here by calculating the amplitudes using LBM from frequency diagrams generated by using block sizes of 33,50 , and 100 ions. The three block sizes used are often found in previous works (Danoix et al., 1992b; Hyde et al., 1995; Read \& Murakami, 1996), and it should also be noted that small changes in block size can give significant changes in appearance of the frequency diagrams (Thuvander et al., 1998).

\section{Results}

\section{Wavelength}

Figure 2 shows the RDFs of $\mathrm{Cr}-\mathrm{Cr}$ in the three aged $\mathrm{Fe}-\mathrm{Cr}$ alloys. The evolution of the RDF with prolonged aging is clearly seen, and it is also compared with the RDF of unaged samples.

When spinodal decomposition is well developed, the wavelength can be read directly from the RDF curve, as
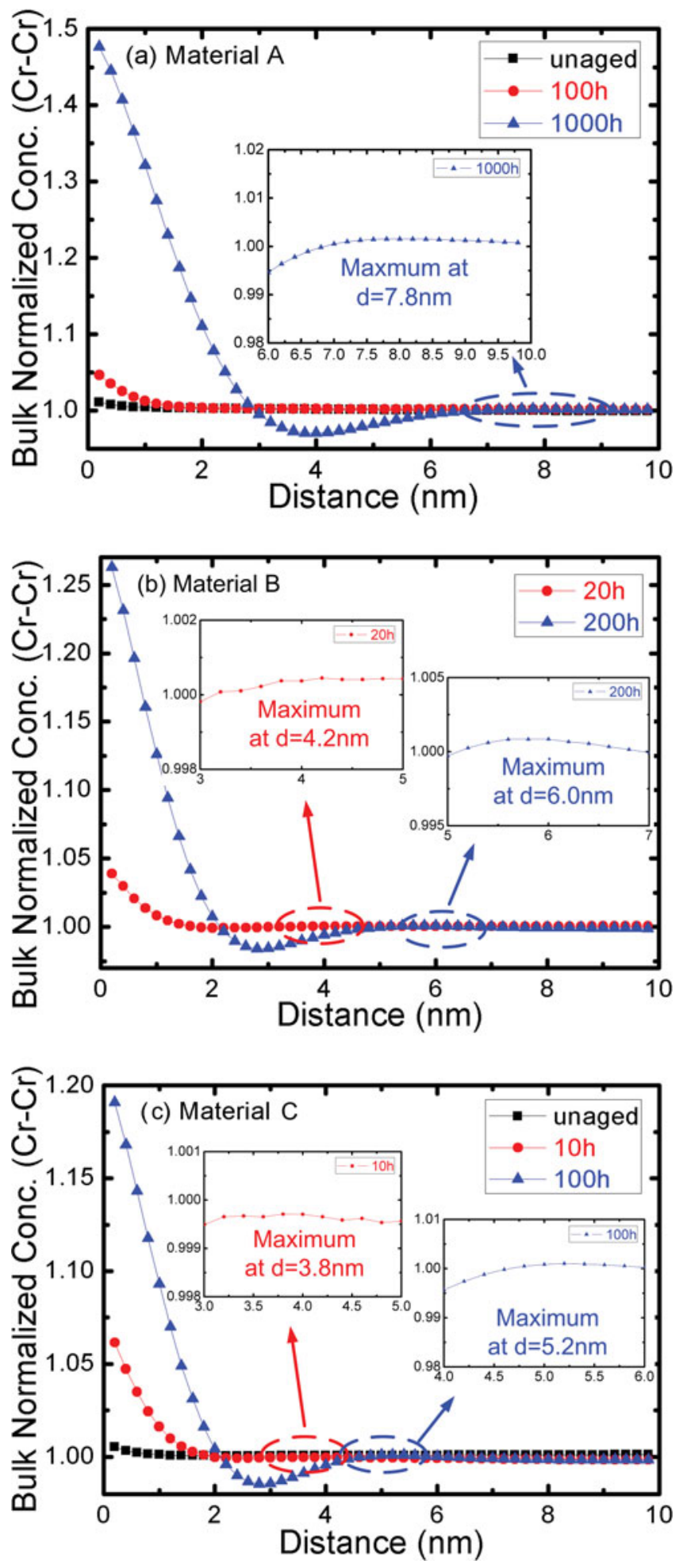

Figure 2. RDFs of $\mathrm{Cr}-\mathrm{Cr}$ used to evaluate the wavelength for all material conditions except material A, aged for $100 \mathrm{~h}$ at $500^{\circ} \mathrm{C}$.

indicated in Figure 2. However, since material A aged for $100 \mathrm{~h}$ at $500^{\circ} \mathrm{C}$ is less decomposed than the other aged samples, no maximum can be found for that RDF curve. During spinodal decomposition the content of $\mathrm{Cr}$ in $\mathrm{Cr}$ rich regions increases, while, on the other hand, the content of $\mathrm{Cr}$ in Fe-rich regions decreases. Therefore, as mentioned 


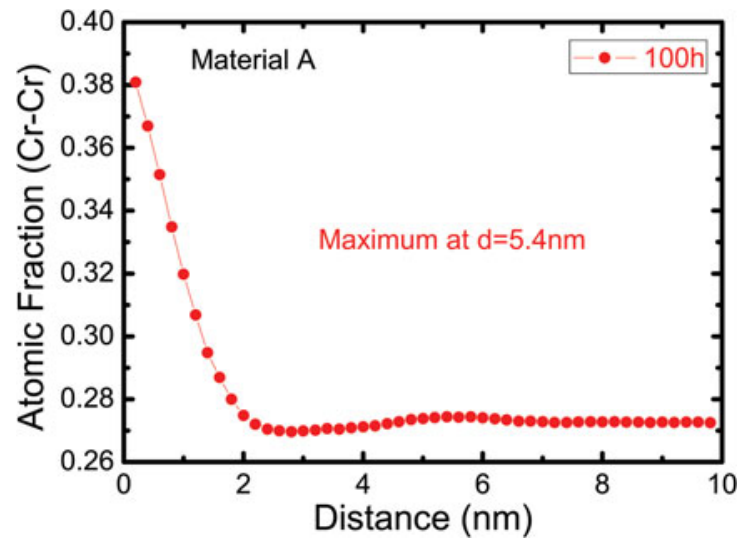

Figure 3. Enhanced RDF of $\mathrm{Cr}-\mathrm{Cr}$ used to evaluate the wavelength for material $\mathrm{A}$, aged for $100 \mathrm{~h}$ at $500^{\circ} \mathrm{C}$.

in the Radial Distribution Function Analysis section, if the $\mathrm{Cr}$ atoms from one Cr-rich region are chosen to generate the RDF curve, there will be an obvious positive interaction at short distances due to the fact that the concentration of $\mathrm{Cr}$ is high in the Cr-rich region. When the distance extends to the nearest neighboring Cr-rich regions, i.e., the distance equaling the wavelength, there exists a maximum on the $\mathrm{RDF}$ curve. Conversely, if the $\mathrm{Cr}$ atoms from one Fe-rich region are chosen as the center, the RDF curve has a negative interaction at short distances. Similarly, a minimum can be found on the RDF curve. It should be noted that although the curves with positive interaction and those with negative interaction destructively interfere, the final RDF curve still has positive interactions at short distances considering that the $\mathrm{Cr}$ atoms in the Cr-rich regions contribute more than $\mathrm{Cr}$ in the $\mathrm{Fe}$-rich regions because the number of $\mathrm{Cr}$ atoms in $\mathrm{Cr}$-rich regions is much larger.

An enhanced RDF was produced for material A, aged for $100 \mathrm{~h}$. It was generated by selecting the $\mathrm{Cr}$ richest domains by iso-concentration surfaces, and hence $100 \mathrm{Cr}-$ rich domains distributed in the whole sample volume were chosen. Cr-Cr RDFs were generated from each Cr-rich domain by starting from each $\mathrm{Cr}$ atom located within a box of $1 \mathrm{~nm} \times 1 \mathrm{~nm} \times 1 \mathrm{~nm}$ at the center of the domain. The 100 RDFs were subsequently averaged, and the enhanced $\mathrm{RDF}$ is presented in Figure 3. It should be noted that the exact number of $\mathrm{Cr}$-rich domains selected is not very important, since it is just a way of generating the RDF from some Cr-rich centers and thus suppressing the effect of Fe-rich areas. The first maximum was then found at $d=5.4 \mathrm{~nm}$ for material A aged for $100 \mathrm{~h}$ at $500^{\circ} \mathrm{C}$ (Fig. 3).

The results from ACF analysis are presented in Figure 4 , and the first maximum denotes the wavelength of

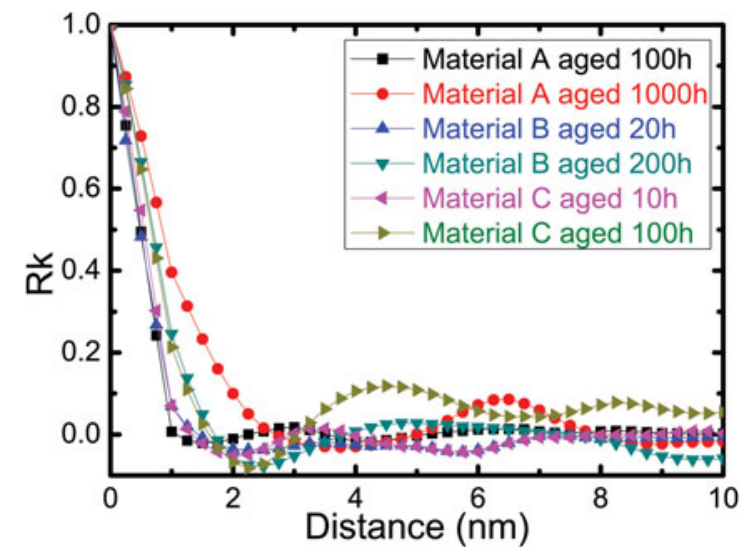

Figure 4. $R_{k}$ values from ACF analysis to evaluate the wavelength for all material conditions.

spinodal decomposition. The wavelengths from ACF and RDF are compiled in Table 2.

\section{Amplitude}

The unaged samples have a very small positive interaction at short distances that already may indicate a small decomposition after quenching from $1100^{\circ} \mathrm{C}$ (Thuvander et al., 2012). However, no wavelength could be determined for the unaged samples, and thus no amplitude was evaluated using $\mathrm{RDF}$. The RDF curves for all samples are presented in Figures 5, 6, and 7 for materials A, B, and C, respectively. The amplitudes for materials $\mathrm{A}, \mathrm{B}$, and $\mathrm{C}$ aged for different times are listed in Table 3. Furthermore, the amplitudes obtained by equation (6), which is based on the zero distance of the RDF, are listed in Table 3, and it should be noted that they are in excellent agreement with the amplitudes obtained from the RDF analysis, except for material C.

The frequency diagrams generated using a block size of 50 ions and LBM fitting are shown in Figures $8 \mathrm{a}, 8 \mathrm{~b}$, and $8 \mathrm{c}$. Furthermore, the amplitudes obtained using the LBM method [equation (2)] using block sizes of 33, 50, and 100 ions are listed in Table 4. In addition, the average compositions of $\mathrm{Cr}$-rich regions and Fe-rich regions, i.e., regions containing more than the nominal concentration of the respective elements, were calculated. Subsequently, the difference between these averages was calculated and is presented in Table 4 under the name Aver*. The sequence of amplitudes is consistent with the results from $\mathrm{RDF}-$ i.e., $\mathrm{A}$, $1000 \mathrm{~h} ; \mathrm{B}, 200 \mathrm{~h}$; C, $100 \mathrm{~h}$; C, 10 h; B, $20 \mathrm{~h}$; and A, $100 \mathrm{~h}$-in order from the most to the least decomposed. The amplitudes obtained by LBM decreases by almost a factor of 2 as the block size increases from 33 to 100 ions. This has also

Table 2. Wavelengths (nm) Obtained for All Material Conditions Using ACF and RDF Analysis.

\begin{tabular}{|c|c|c|c|c|c|c|}
\hline & \multicolumn{2}{|c|}{ Material A } & \multicolumn{2}{|c|}{ Material B } & \multicolumn{2}{|c|}{ Material C } \\
\hline & Aged $100 \mathrm{~h}$ & Aged $1000 \mathrm{~h}$ & Aged $20 \mathrm{~h}$ & Aged $200 \mathrm{~h}$ & Aged $10 \mathrm{~h}$ & Aged $100 \mathrm{~h}$ \\
\hline $\mathrm{RDF}$ & 5.4 & 7.8 & 4.2 & 6.0 & 3.8 & 5.2 \\
\hline $\mathrm{ACF}$ & 3 & 6.5 & 3.5 & 4.75 & 3.5 & 4.5 \\
\hline
\end{tabular}



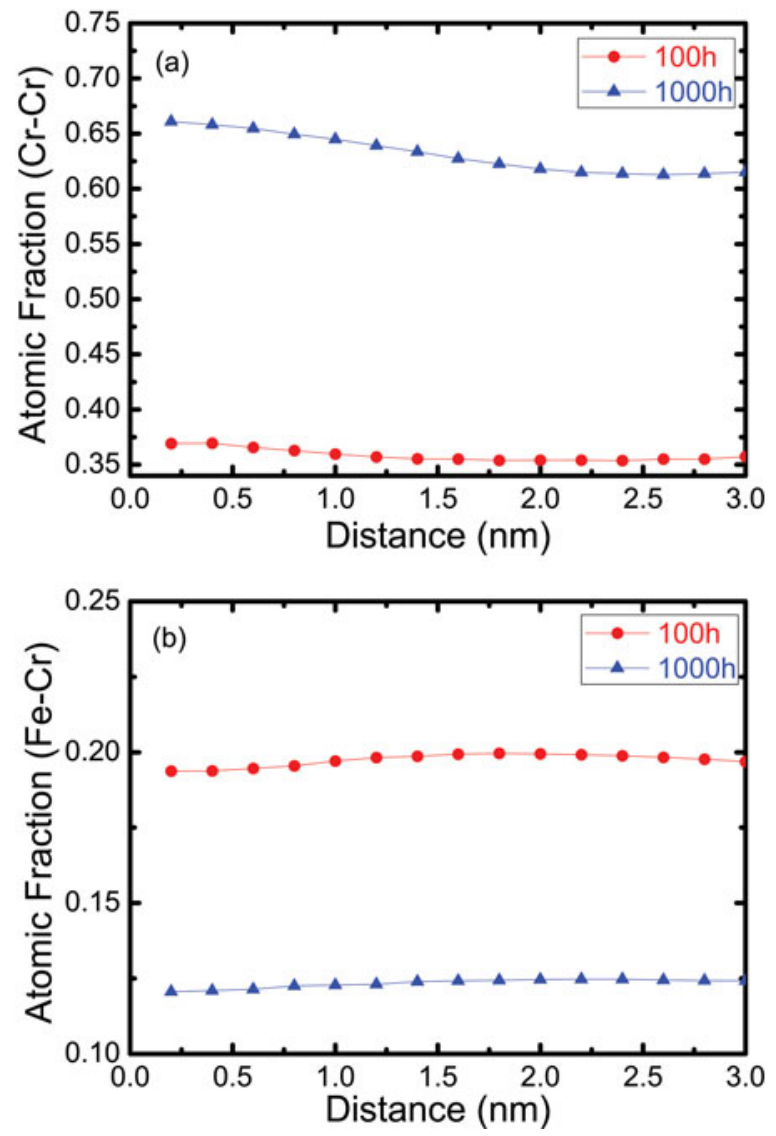

Figure 5. a: RDF of Cr-Cr in Cr-rich regions for material A, aged for $100 \mathrm{~h}$ and $1000 \mathrm{~h}$. b: RDF of Fe-Cr in Fe-rich regions for material A, aged for $100 \mathrm{~h}$ and $1000 \mathrm{~h}$.

been explained previously in Hyde et al. (1995) because a small block size cannot smooth out the noise while a large block size will smooth over the composition fluctuations.

\section{Discussion}

\section{Wavelength}

Wavelengths for the different material conditions obtained using both RDF and 1D ACF are compared in Figure 9. The wavelengths from ACF, as well as from previous work using 1D FFT in Odqvist et al. (2012), are shorter than those from RDF for all cases. It should be remembered that any $1 \mathrm{D}$ method is sensitive to anisotropy. Although the 3D reconstruction was conducted so that the volume would appear isotropic, small differences in decomposition in the different directions would immediately appear in the 1D methods. The RDF is better at describing the $3 \mathrm{D}$ decomposition and is not as sensitive to anisotropy or small reconstruction errors. It should also be noted that the ACF can be performed in 3D, and it is predicted to give similar results as the RDF because they are related. The difference between $1 \mathrm{D}$ ACF and RDF is largest for material A, aged for $100 \mathrm{~h}$, which shows the lowest decomposition. It is likely that the low statistics in the ACF gives the rather large difference as compared to RDF. Thus, it is especially impor-
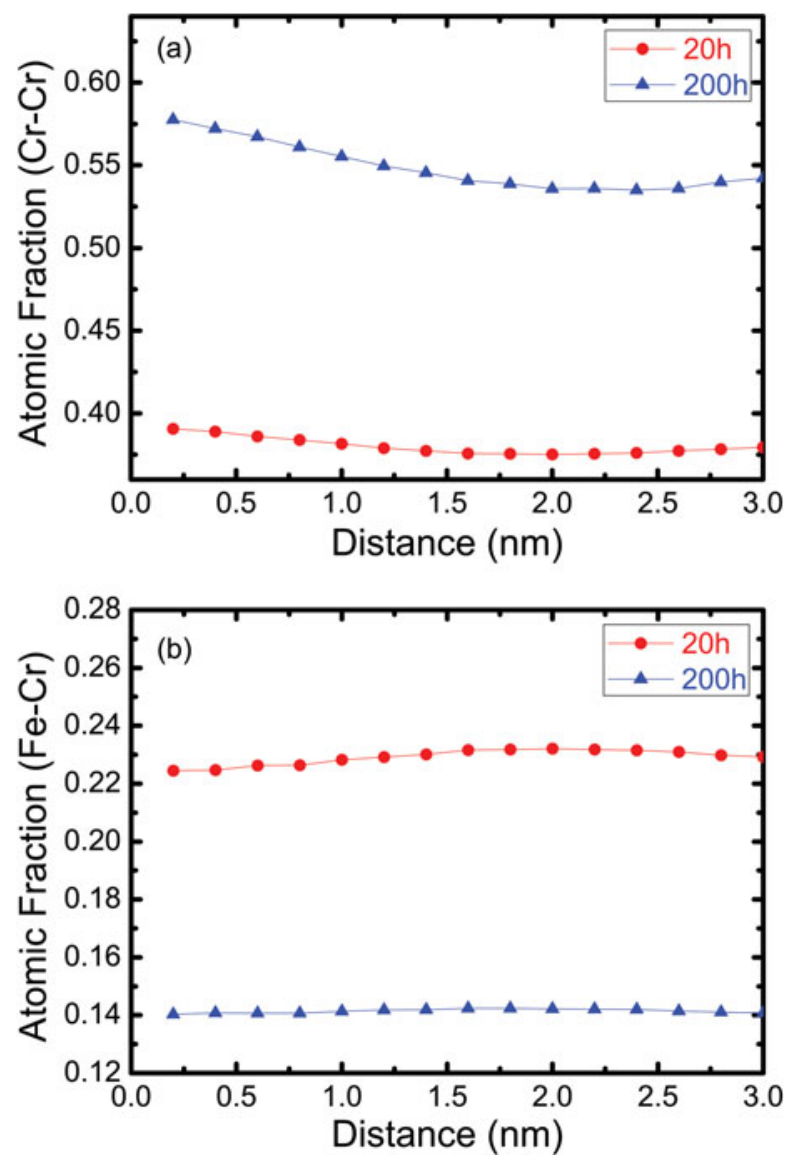

Figure 6. a: $\mathrm{RDF}$ of $\mathrm{Cr}-\mathrm{Cr}$ in $\mathrm{Cr}-$ rich regions for material $\mathrm{B}$ aged for $20 \mathrm{~h}$ and $200 \mathrm{~h}$. b: RDF of $\mathrm{Fe}-\mathrm{Cr}$ in Fe-rich regions for material $\mathrm{B}$ aged for $20 \mathrm{~h}$ and $200 \mathrm{~h}$.

tant to use the full 3D data when the decomposition is in the early stages. It should also be noted that material A was claimed to be located in a transient region between nucleation and growth and spinodal decomposition in earlier works by some of the present authors (Xiong et al., 2011; Hedström et al., 2012). It is still our belief that material A is in the transient region and that both mechanisms of decomposition are active, but a similar analysis method was used for this material condition and it can be seen that the wavelength of decomposition increases, although the amplitude is not fully developed. This could indicate that the present analysis method is also suitable to characterize the nanostructure in this case.

Estimation of the wavelength is critical, especially when it comes to the comparison between experiments and modeling. A small error in calculation of the diffusion distance would lead to considerable errors with respect to spinodal decomposition kinetics. Therefore, a theoretical prediction of the dominant wavelength based on the latest thermodynamics description (Xiong et al., 2011) was attempted. According to Cahn's linearized theory of spinodal decomposition (Cahn, 1961), which should hold reasonably well at early stages, the wavelength of the fastest growing wave is given by $\lambda_{\max }=\sqrt{2} \lambda_{c}$, where $\lambda_{c}$ is the critical wavelength given by 

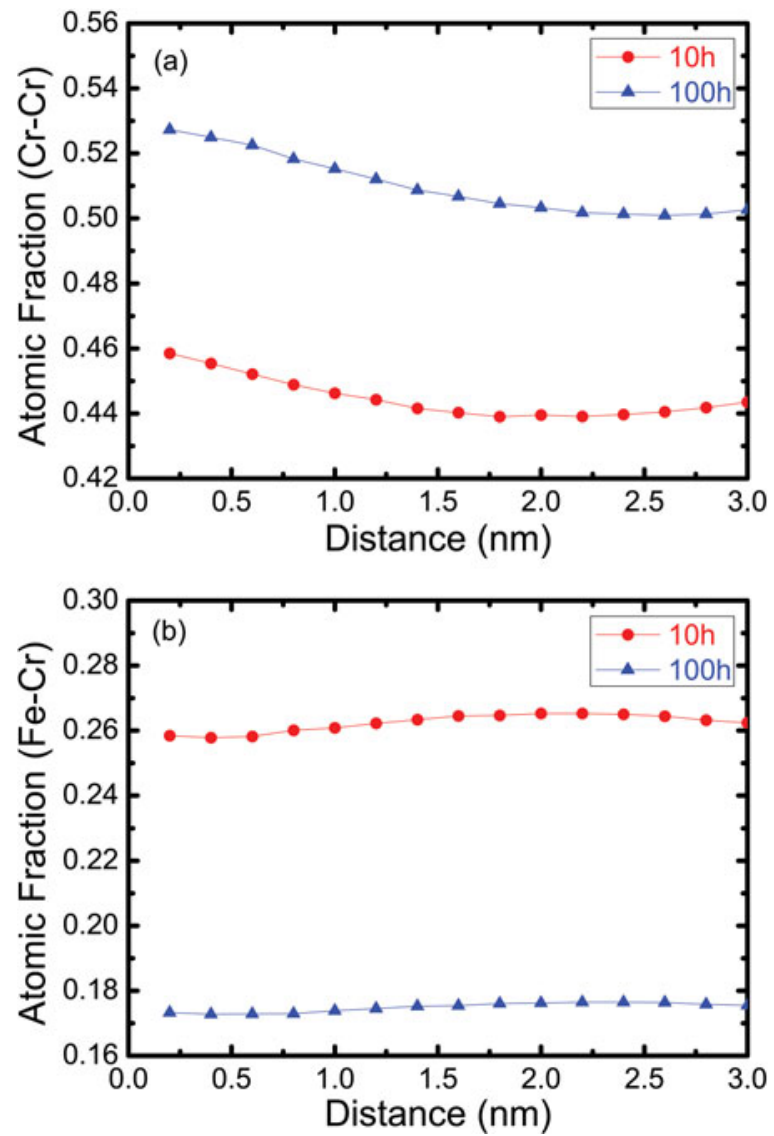

Figure 7. a: $\mathrm{RDF}$ of $\mathrm{Cr}-\mathrm{Cr}$ in $\mathrm{Cr}$-rich regions for material $\mathrm{C}$ aged for $10 \mathrm{~h}$ and $100 \mathrm{~h}$. b: RDF of Fe-Cr in Fe-rich regions for material $\mathrm{C}$ aged for $10 \mathrm{~h}$ and $100 \mathrm{~h}$.

$$
\lambda_{c}=2 \pi \sqrt{-\frac{2 \epsilon}{d^{2} G_{m} / d x_{C r}^{2}}} .
$$

In equation (8) the gradient energy coefficient $(\epsilon)$ is expressed in terms of nearest neighbor interactions and the interaction length (Cahn, 1961). For the present case the interaction energy is taken as the regular solution parameter evaluated by Xiong et al. (2011), and the interaction length is put to $2.5 \AA$. The fastest growing wavelength for materials $\mathrm{B}(20 \mathrm{~h})$ and $\mathrm{C}(10 \mathrm{~h})$ is $2.8 \mathrm{~nm}$ and $1.2 \mathrm{~nm}$, respectively. Compared to the wavelengths from RDF, the calculated values (see Table 2) are much lower. One reason for this is the uncertainty in the gradient energy coefficient, e.g., the effect of magnetism is not accounted for in the regular solution parameter.

The wavelengths obtained from RDF in Figure 9 show a power-law behavior:

$$
\lambda \propto t^{\alpha}
$$

where $\lambda$ is the wavelength of spinodal decomposition, $t$ is the aging time, and $\alpha$ is the exponent of $t$.

The value of $\alpha$ in equation (9) is estimated to about 0.16 for all three alloys, though it should be noted that the value is somewhat uncertain because only two aging times were used for each material. The value of 0.16 is still in
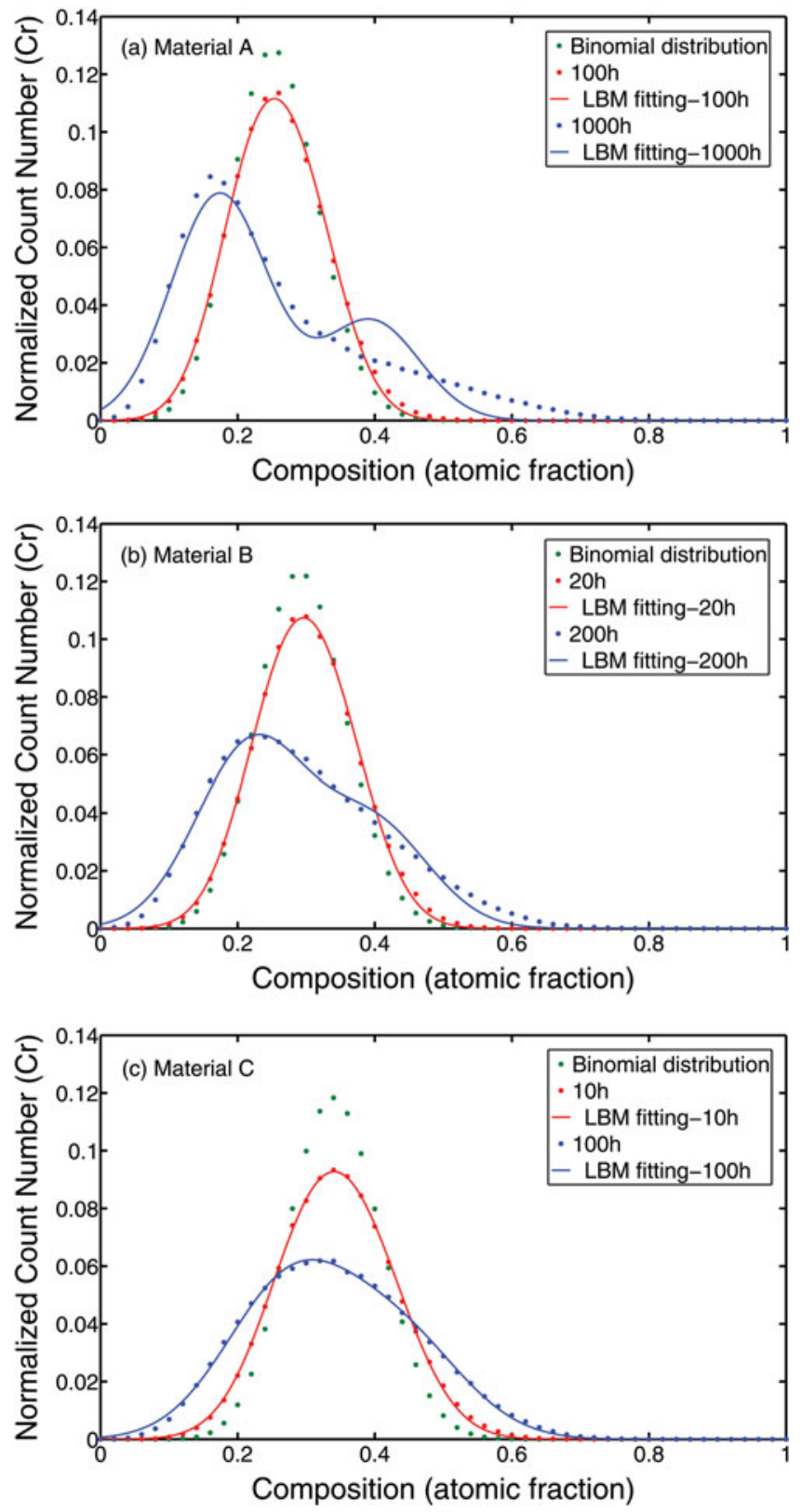

Figure 8. Frequency diagrams of $\mathrm{Cr}$ distribution generated using a block size of 50 atoms together with the binomial distribution curves and the LBM fitting results. a: Material A aged for $100 \mathrm{~h}$ and 1000 h. b: Material B aged for 20 h and 200 h. c: Material C aged for $10 \mathrm{~h}$ and $100 \mathrm{~h}$.

good agreement with previous works, including Monte Carlo simulations: $\alpha=0.17-0.25$ (Rao et al., 1976; Lebowitz et al., 1982; Grest \& Srolovitz, 1984; Hyde et al., 1995); experimental results: $\alpha=0.2$ (Blavette \& Auger, 1990), $\alpha=0.18$ (Danoix et al., 1992a), $\alpha=0.21$ (Hyde et al., 1995), and $\alpha=$ 0.16 (Pareige et al., 2011); and a theoretical prediction of about 0.18 (Langer et al., 1975). The agreement between the RDF method and the previous works on wavelength evolution strengthens the view that the RDF method is suitable for quantifying spinodal decomposition. On the other hand, the wavelengths obtained using 1D ACF have no clear power-law behavior. It should be noted that Huse (1986) 
Table 3. Peak, Trough, and Amplitudes Obtained Using RDF Analysis on All Material Conditions.

\begin{tabular}{lcccccccc}
\hline & \multicolumn{2}{c}{ Material A } & & \multicolumn{2}{c}{ Material B } & & \multicolumn{2}{c}{ Material C } \\
\cline { 2 - 3 } & $100 \mathrm{~h}$ & $1000 \mathrm{~h}$ & & $20 \mathrm{~h}$ & $200 \mathrm{~h}$ & & $10 \mathrm{~h}$ & $100 \mathrm{~h}$ \\
\hline Peak value (\%) & 36.9 & 66.1 & & 39.1 & 57.8 & & 45.9 & 52.7 \\
Trough value (\%) & 19.4 & 12.1 & & 22.5 & 14.0 & & 25.8 & 17.3 \\
Amplitude (\%) & 17.5 & 54.0 & & 16.6 & 43.8 & & 20.1 & 35.4 \\
Amplitude from equation (7) (\%) & 17.7 & 52.9 & & 18.8 & 46.9 & & 28.1 & 46.5 \\
\hline
\end{tabular}

Table 4. Amplitudes Obtained Using the LBM Method Together with the Average Compositions of Cr-Rich and Fe-Rich Regions.

\begin{tabular}{|c|c|c|c|c|c|c|}
\hline Material & $\begin{array}{l}\text { Aging } \\
\text { Time }\end{array}$ & & $\begin{array}{l}\text { Block Size } \\
\text { (ions) }\end{array}$ & $\begin{array}{c}\text { Peak Value } \\
(\%)\end{array}$ & $\begin{array}{c}\text { Trough Value } \\
(\%)\end{array}$ & $\begin{array}{l}\text { Amplitude } \\
(\%)\end{array}$ \\
\hline \multirow[t]{8}{*}{ Material A } & $100 \mathrm{~h}$ & LBM & 33 & 31.3 & 21.8 & 9.5 \\
\hline & & & 50 & 30.1 & 22.5 & 7.6 \\
\hline & & & 100 & 28.7 & 23.5 & 5.2 \\
\hline & & Aver ${ }^{*}$ & & 29.0 & 23.6 & 5.4 \\
\hline & $1000 \mathrm{~h}$ & LBM & 33 & 40.5 & 17.1 & 23.4 \\
\hline & & & 50 & 39.4 & 17.4 & 22.0 \\
\hline & & & 100 & 36.7 & 17.7 & 19.0 \\
\hline & & Aver ${ }^{*}$ & & 38.6 & 16.9 & 21.7 \\
\hline \multirow[t]{8}{*}{ Material B } & $20 \mathrm{~h}$ & LBM & 33 & 35.1 & 25.8 & 9.3 \\
\hline & & & 50 & 34.0 & 26.6 & 7.4 \\
\hline & & & 100 & 32.6 & 27.7 & 4.9 \\
\hline & & Aver $^{*}$ & & 34.7 & 27.6 & 7.1 \\
\hline & $200 \mathrm{~h}$ & LBM & 33 & 40.9 & 21.7 & 19.2 \\
\hline & & & 50 & 39.9 & 22.1 & 17.8 \\
\hline & & & 100 & 37.9 & 22.8 & 15.1 \\
\hline & & Aver ${ }^{*}$ & & 39.0 & 21.1 & 17.9 \\
\hline \multirow[t]{8}{*}{ Material C } & $10 \mathrm{~h}$ & LBM & 33 & 40.5 & 29.8 & 10.7 \\
\hline & & & 50 & 30.6 & 39.2 & 8.6 \\
\hline & & & 100 & 37.9 & 31.6 & 6.3 \\
\hline & & Aver ${ }^{*}$ & & 40.0 & 30.6 & 9.4 \\
\hline & $100 \mathrm{~h}$ & LBM & 33 & 44.8 & 27.1 & 17.7 \\
\hline & & & 50 & 43.8 & 27.6 & 16.2 \\
\hline & & & 100 & 42.0 & 28.3 & 13.7 \\
\hline & & Aver* & & 43.7 & 25.8 & 17.9 \\
\hline
\end{tabular}

claimed that the exponent value changes with time because the diffusion pathways evolve with aging time in late stages of spinodal decomposition. However, in the present case the decomposition is rather early for all sample conditions.

\section{Amplitude}

All of the results for amplitudes, including the calculated Aver ${ }^{\star}$, are shown in Figure 10. It should be noted that the amplitudes obtained by the LBM method coincide with the Aver* values. This is a clear indication that the LBM method is seriously underestimating the amplitude of spinodal decomposition. As stated in the Amplitude subsection in the Results section, the Aver* value gives the difference between the average of Cr-rich (above average Cr concentration) and Fe-rich (above average $\mathrm{Fe}$ concentration) regions, i.e., the Aver ${ }^{\star}$ is not representative for the peak and trough. The
LBM method separates the distribution curve into two Gaussian curves, which represent $\alpha$ and $\alpha^{\prime}$ with Cr compositions below and above the average $\mathrm{Cr}$ concentration, respectively. The two peaks represent the largest possibility to find this $\mathrm{Cr}$ concentration in the Cr-rich regions and the Fe-rich regions, which is more related to the average $\mathrm{Cr}$ concentration of $\alpha$ and $\alpha^{\prime}$ than the peak and trough values.

Moreover, the LBM method is suggested to be suitable for the early stages of spinodal decomposition when the frequency distribution diagram broadening is symmetrical (Miller et al., 1992). This is in accord with the results in the present work. All distribution diagrams from the alloys after short time aging are rather well represented by two Gaussian curves. In addition, the relatively long-term aging of material $\mathrm{C}$ is also fairly well represented by the two Gaussian curves. This agrees with the theory that the broadening 


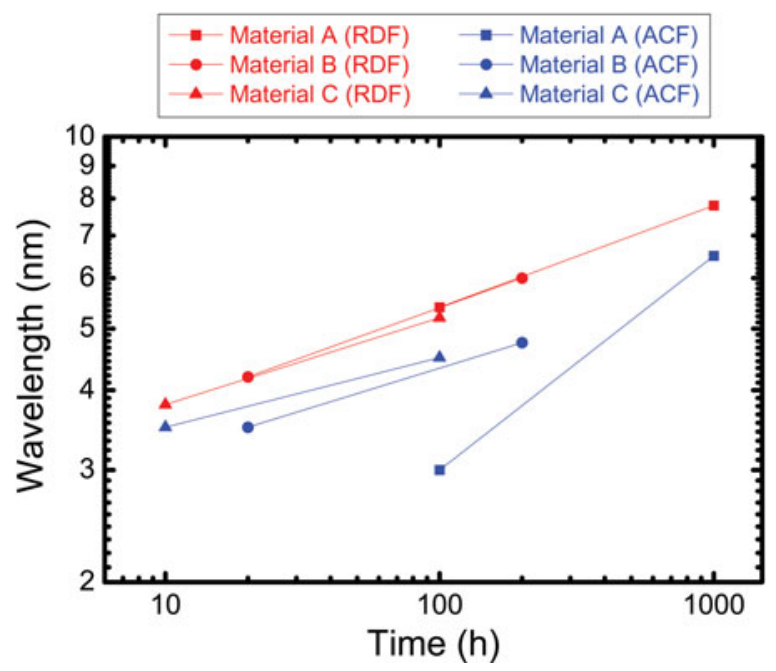

Figure 9. Time evolution of wavelengths obtained using RDF and ACF analyses.

\begin{tabular}{|c|c|}
\hline - Material A (RDF) & - Material A (LBM-50 ions) \\
\hline - Material B (RDF) & - Material B (LBM-50 ions) \\
\hline$\triangle-$ Material C (RDF) & $\triangle-$ Material C (LBM-50 ions) \\
\hline --Material A (Eq. (7)) & - Material A (Aver*) \\
\hline$\rightarrow$ Material B (Eq. (7)) & $\rightarrow$ Material B (Aver*) \\
\hline$\_$Material C (Eq. (7)) & $\triangle-$ Material C (Aver*) \\
\hline
\end{tabular}

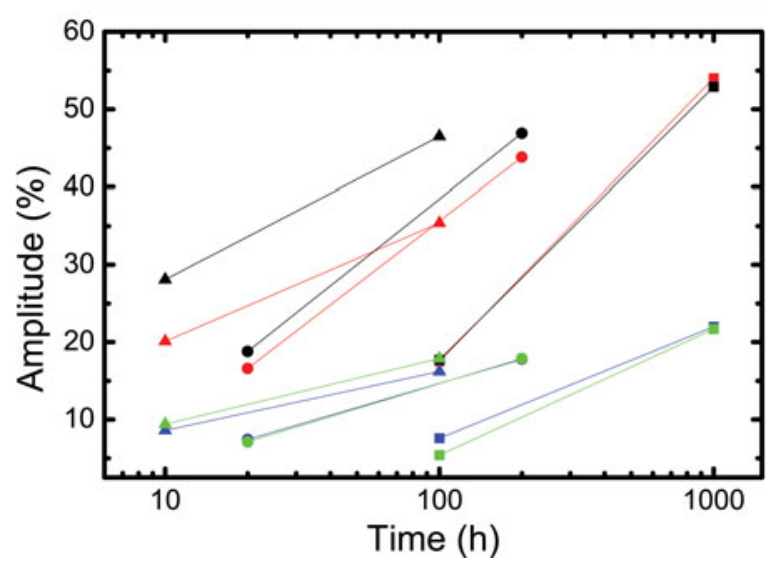

Figure 10. Time evolution of amplitudes obtained using different analysis methods such as RDF, equation (7), LBM, and Aver*.

will be symmetrical in the early stages of decomposition for samples that are rather centered in the miscibility gap such as, for example, $\mathrm{Fe}-45 \% \mathrm{Cr}$. However, the composition of interest in most materials systems-for example, commercial stainless steels or TiAlN coatings-is not centered in the miscibility gap and thus will produce asymmetric broadening even in the rather early stages of decomposition. It is therefore suggested that the RDF method is more suitable to deal with this challenge because it does not depend on symmetry of the frequency diagram.

Amplitudes obtained from RDF analysis are in good agreement with the amplitudes calculated from $\mathrm{RDF}(0)$ and using equation (7). It should be pointed out that equation (7), as deduced in the Radial Distribution Function Analysis section, is a theoretical treatment of the RDF assuming sinusoidal decomposition waves, and it provides a simple means to calculate the amplitude from any RDF curve. This is very promising, especially because equation (7) can also be used for the very early stages of decomposition. For instance, the unaged samples also show a slight positive $\mathrm{Cr}-\mathrm{Cr}$ interaction in the RDF, and using equation (7) the amplitude could be evaluated for these conditions as well. It should be noted that the Cr-Cr RDF from austenite shows no positive interaction, and it is thus believed that the slight positive interaction of $\mathrm{Cr}-\mathrm{Cr}$ in homogenized ferrite is due to phase separation. However, some more work is needed to make sure that this is not some type of artifact from the APT technique.

It can also be noted that in Xiong (2012) the amplitude of material $\mathrm{C}$ aged for $100 \mathrm{~h}$ (same as in the present work) was evaluated using the amplitude density spectrum (ADS) method. There is excellent agreement between the amplitudes found by using $1 \mathrm{D}$ concentration profiles and ADS (Xiong, 2012) and the RDF method in the present work: $34.8 \%$ and $35.4 \%$, respectively. The benefit of the method proposed in the present work, however, is that the utilization of the wavelength determined first gives a robust and well-defined means to quantify the amplitude.

\section{CONCLUSIONS}

- A new method based on RDF analysis to evaluate wavelength and amplitude of spinodal decomposition has been proposed.

- The new method does not depend on the selection of box size to evaluate the concentration.

- The amplitude estimated from RDF analysis is always larger than the amplitude obtained by the LBM method. This is due to an underestimation using the LBM method.

- A theoretical treatment, assuming a sinusoidal concentration variation, of the amplitude from RDF was suggested. Results obtained were similar to the results obtained from the more involved RDF method outlined in this article. Using the theoretical treatment it is possible to determine the amplitude of spinodal decomposition in the very early stages, even when it is not possible to see a statistical difference with the binomial distribution in the frequency diagram.

\section{ACKNOWLEDGMENTS}

This project was supported by the Swedish Research Council (VR, Grant No. 621-2009-5289) and the VINNEX Centre Hero-m, financed by the Swedish Governmental Agency for Innovation Systems (VINNOVA), Swedish industry, and KTH Royal Institute of Technology. J.Z. thanks ERASMUS MUNDUS TANDEM for financial support.

\section{REFERENCES}

Blavette, D. \& Auger, P. (1990). Fine scale investigation of some phenomena in metallic alloys by field ion microscopy and atom probe microanalysis. Microsc Microanal Microstruct 1, 481-492.

Blavette, D., Grancher, G. \& Bostel, A. (1988). Statistical analysis of atom-probe data (I): Derivation of some fine scale 
features from frequency distributions for finely dispersed systems. J de Phys 49(C6), 433-438.

Bley, F. (1992). Neutron small-angle scattering study of unmixing in Fe-Cr alloys. Acta Metall Mater 40, 1505-1517.

Brenner, S.S., Camus, P.P., Miller, M.K. \& Soffa, W.A. (1984). Phase separation and coarsening in Fe-Cr-Co alloys. Acta Metall 32, 1217-1227.

Brenner, S.S., Miller, M.K. \& Soffa, W.A. (1982). Spinodal decomposition of iron-32 at.\% chromium at $470^{\circ} \mathrm{C}$. Scripta Metall 16, 831-836.

Brown, J.E., Cerezo, A., Godfrey, T.J., Hetherington, M.G. \& Sмith, G.D.W. (1990). Quantitative atom probe analysis of spinodal reaction in ferrite phase of duplex stainless steel. Mater Sci Tech 6, 293-300.

Brown, J.E. \& Sмith, G.D.W. (1991). Atom probe study of spinodal processes in duplex stainless steels and in single- and dual-phase Fe-Cr-Ni alloys. Surf Sci 246, 285-291.

Cahn, J.W. (1961). On spinodal decomposition. Acta Metall 9, 795-801.

Cahn, J.W. (1968). Spinodal decomposition. Trans AIME 242, $166-180$.

CAhn, J.W. \& Hilliard, J.E. (1958). Free energy of a nonuniform system: I. J Chem Phys 28, 258-267.

Chandra, D. \& Schwartz, L.H. (1971). Mössbauer effect study of the $475^{\circ} \mathrm{C}$ decomposition of Fe-Cr. Metall Trans 2, 511-519.

Chung, H.M. \& Chopra, O.K. (1988). Kinetics and mechanism of thermal aging embrittlement of duplex stainless steels. Proceedings of the 3rd International Symposium on Environmental Degradation of Materials in Nuclear Power Systems-Water Reactors, pp. 359-370. Warrendale, PA: Metallurgical Society Inc.

Chung, H.M. \& Leax, T.R. (1990). Embrittlement of laboratory and reactor aged CF3, CF8, and CF8M duplex stainless steels. Mater Sci Tech 6, 249-262.

Danoix, F., Auger, P. \& Blavette, D. (1992a). An atom-probe investigation of some correlated phase transformations in $\mathrm{Cr}$, Ni, Mo containing supersaturated ferrites. Surf Sci 266, 364-369.

Danoix, F., Deconihout, B., Bostel, A. \& Auger, P. (1992b). Some new aspects on microstructural and morphological evolution of thermally aged duplex stainless steels. Surf Sci 266, 409-415.

De Geuser, F., Lefebvre, W. \& Blavette, D. (2006). 3D atom probe study of solute atoms clustering during natural ageing and pre-ageing of an Al-Mg-Si alloy. Philos Mag Lett 86, 227-234.

Gault, B., De Geuser, F., Stephenson, L.T., Moody, M.P., MudDLE, B.C. \& RingeR, S.P. (2008). Estimation of the reconstruction parameters for atom probe tomography. Microsc Microanal 14, 296-305.

Godfrey, T.J., Hetherington, M.G., Sassen, J.M. \& Smith, G.D.W. (1988). The characterization of spinodal structures in duplex CF3 steels. J de Phys 49, 421-426.

Grest, G.S. \& Srolovitz, J. (1984). Structure and evolution of quenched Ising clusters. Phys Rev B 30, 5150-5155.

Hedström, P., Baghsheikhi, S., Liu, P. \& Odqvist, J. (2012). A phase-field and electron microscopy study of phase separation in Fe-Cr alloys. Mater Sci Eng A 534, 552-556.

Hetherington, M.G. \& Miller, M.K. (1989). Some aspects of the measurement of composition in the atom probe. Colloq Physique 50, 535-540.

Hillert, M. (1956). A theory of nucleation for solid metallic solutions. PhD Thesis. Cambridge, MA: Massachusetts Institute of Technology.
Hillert, M. (1961). A solid-solution model for inhomogeneous systems. Acta Metall 9, 525-535.

Hörling, A., Hultman, L., Oden, M., Sjölen, J. \& Karlsson, L. (2005). Mechanical properties and machining performance of $\mathrm{Ti}_{1-x} \mathrm{Al}_{x} \mathrm{~N}$-coated cutting tools. Surf Coat Tech 191, 384-392.

Huse, D.A. (1986). Corrections to late-stage behavior in spinodal decomposition: Lifshitz-Slyozov scaling and Monte Carlo simulations. Phys Rev B 34, 7845-7850.

Hyde, J.M., Cerezo, A., Miller, M.K. \& Smith, G.D.W. (1994). A study of the effect of ageing temperature on pahse separation in Fe-45\%Cr alloys. Appl Surf Sci 76/77, 233-241.

Hyde, J.M., Miller, M.K., Hetherington, M.G., Cerezo, A., Sмith, G.D.W. \& Elliott, C.M. (1995). Spinodal decomposition in Fe-Cr alloys: Experimental study at the atomic level and comparison with computer models-II. Development of domain size and composition amplitude. Acta Metall Mater 43, 3404-3413.

Langer, J.S., Baron, M. \& Miller, H.D. (1975). New computational method in the theory of spinodal decomposition. Phys Rev A 11, 1417-1429.

Lebowitz, J.L., Marro, J. \& Kalos, M.H. (1982). Dynamical scaling of structure function in quenched binary alloys. Acta Metall 30, 297-310.

Miller, M.K. (2000). Atom Probe Tomography: Analysis at the Atomic Level. New York: Kluwer Academic/Plenum Publishers.

Miller, M.K., Brenner, S.S., Camus, P.P., Piller, J. \& Soffa, W.A. (1982). Low-temperature precipitation in iron-chromium binary alloys. Proc Int Field Emiss Symp 29, 489-496.

Miller, M.K., Cerezo, A., Hetherington, M.G \& Hyde, J.M. (1992). Estimation of composition amplitude: $P_{a}$ and LBM versus V. Surf Sci 266, 446-452.

Miller, M.K., Hyde, J.M., Hetherington, M.G., Cerezo, A., Sмith, G.D.W. \& Elliotт, C.M. (1995). Spinodal decomposition in Fe-Cr alloys: Experimental study at the atomic level and comparison with computer models-I. Introduction and methodology. Acta Metall Mater 43, 3385-3401.

Miller, M.K. \& KeniK, E.A. (2004). Atom probe tomography: A technique for nanoscale characterization. Microsc Microanal 10, 336-341.

Odqvist, J., Zhou, J., Xiong, W., Hedström, P., Thuvander, M., Selleby, M. \& Ågren, J. (2012). 3D analysis of phase separation in ferritic stainless steels. In Proceedings 3D Material Science 2012. Pittsburgh, PA: TMS.

Oehring, M. \& Alvensleben, L.V. (1988). Evaluation of atom probe concentration profiles by autocorrelation analysis. $J$ de Phys 49, 415-420.

Okada, M., Thomas, G., Нomma, M. \& Kaneko, H. (1978). Microstructure and magnetic properties of $\mathrm{Fe}-\mathrm{Cr}-\mathrm{Co}$ alloys. IEEE Trans Mag 14, 245-252.

Pareige, C., Novy, S., Saillet, S. \& Pareige, P. (2011). Study of phase transformation and mechanical properties evolution of duplex stainless steels after long term thermal ageing $(>20$ years). J Nucl Mater 411, 90-96.

Rao, M., Kalos, M.H., Lebowitz, J.L. \& Marro, J. (1976). Time evolution of a quenched binary alloy. III. Computer simulation of a two-dimensional model system. Phys Rev B 13, 4328-4335.

Read, H.G. \& Murakami, H. (1996). Microstructural influences on the decomposition of an Al-containing ferritic stainless steel. App Surf Sci 94/95, 334-342.

Sassen, J.M., Hetherington, M.G., Godfrey, T.J., Smith, G.D.W., Pumphrey, P.H. \& Akhurst, K.N. (1987). Kinetics of spinodal reaction in the ferrite phase of a duplex stainless steel. In 
Properties of Stainless Steels in Elevated Temperature Service, Prager, M. (Ed.), pp. 65-78. New York: AMSE.

Thuvander, M., Andrèn, H.-O., Stiller, K. \& Hu, Q.H. (1998). A statistical method to detect ordering and phase separation by APFIM. Ultramicroscopy 73, 279-285.

Thuvander, M., Zhou, J., Odqvist, J., Hertzman, S. \& HedSTRÖM, P. (2012). Observations of copper clustering in a 25Cr7Ni super duplex stainless steel during low-temperature aging under load. Phil Mag Lett 92, 336-343.

XIOng, W. (2012). Thermodynamic and kinetic investigation of the Fe-Cr-Ni system driven by engineering applications. $\mathrm{PhD}$ Thesis. Sweden: KTH.
Xiong, W., Hedström, P., Selleby, M., Odqvist, J., Thuvander, M. \& CHen, Q. (2011). An improved thermodynamic modeling of the Fe-Cr system down to zero kelvin coupled with key experiments. CALPHAD 35, 355-366.

Zhou, J., Odqvist, J., Thuvander, M., Hertzman, S. \& Hedsтröм, P. (2012). Concurrent phase separation and clustering in the ferrite phase during low temperature stress-aging of duplex stainless steel weldments. Acta Mater 60, 5818-5827. 